\title{
Virtual Reality and Beyond: The LabTEVE at UFPB in Brazil
}

\author{
Liliane S. Machado and Ronei M. Moraes \\ Laboratory of Technologies for Virtual Teaching and Statistics (LabTEVE) \\ http://www.de.ufpb.br/ labteve \\ Federal University of Paraíba (UFPB) \\ Cidade Universitária s/n - 58051-900 - João Pessoa, Brazil \\ liliane@di.ufpb.br,ronei@de.ufpb.br
}

\begin{abstract}
Training systems, serious games, assessment methods, solution for VR and systems to support learning have been the focus of research at LabTEVE. Since 2000 several projects have been developed and evidenced the interdisciplinary context of the group researches. This paper presents some past and present activities developed in the LabTEVE at UFPB in Brazil and points to future trends.
\end{abstract}

Keywords-training systems, virtual learning, assessment methods, serious games, virtual reality.

\section{INTRODUCTION}

The use of immersive and interactive tools to support learning and teaching has influenced the society and promoted new ways to deal with computer applications. Threedimensional (3D) visualization, interactive touch, tracking and intelligent monitoring permeate several human activities and can be related to virtual reality (VR) researches. In Brazil, at Federal University of Paraíba (UFPB), the Laboratory of Technologies for Virtual Teaching and Statistics (LabTEVE) was created in 2000 with the mission of development and integration of technologies for virtual teaching and distance learning. The interdisciplinary focus of the researches allows the integration of several areas to produce innovative solutions.

In this paper are presented the main research areas of LabTEVE, some past and present projects, and main directions to be followed in future.

\section{Main Research Areas}

It is know that the VR can permeate several research areas, as Computer Sciences, Statistics, Engineering, human physiology, Psychology, Mathematics and many others. Therefore, the integration of interdisciplinary teams is a central point of many LabTEVE projects. It includes the development of medical simulators for training, interactive visualization applications, visualization platforms, games, collaborative applications and tools for programming.

The main research areas of LabTEVE can be grouped in five categories to present the first developments and the ongoing projects: solutions for VR, systems to support learning, VR applications development, serious games and assessment.

\section{SOLUTIONS FOR VR}

LabTEVE has developed low cost systems solutions for VR visualization and 3D interaction. Since 2002, new platforms have been integrated to solve problems related to the cost and mobility of immersive systems presenting an accessible solution for Brazilian companies and universities for several applications. Three different solutions offer systems for immersive and interactive visualization for multiple users. All these solutions are based on a single commodity computer and multimedia projectors: the VirtWall ${ }^{\mathrm{TM}}$ (Figure 1), which is a wall based on a single computer (2002), the Biface system (two walls, developed in 2009) and the Triface system (three walls, developed in 2011). All these solutions have patent required.

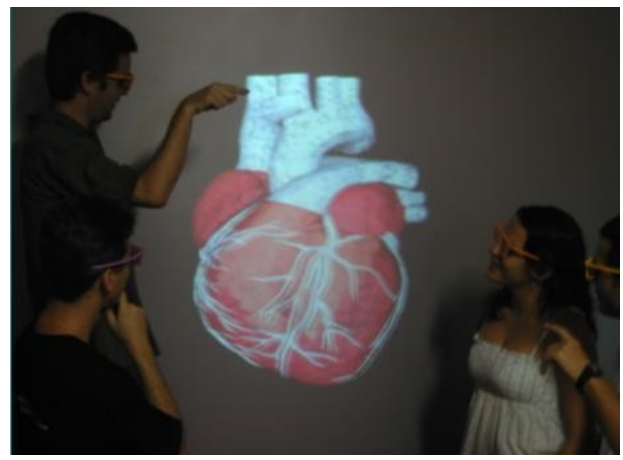

Figure 1: VirtWall platform in use.

In order to solve problems and difficulties related to the integration of VR systems for medical purposes, the CyberMed project was started in 2004 to produce a framework to support the reuse of components and rapid integration of simulators. The CyberMed 1.0 included 3D visualization techniques, interaction with haptics, collision detection and interactive deformations methods, mesh import and user assessment methods [1]. The framework was registered and made available for free download at SourceForge in 2007. Since then, the project continued to be expanded and the 2.0 version included support to magnetic and optical tracking [2], tutoring and collaboration activities, new haptic and interaction devices, new assessment methods and installation scripts (Figure 2) [3]. Nowadays, CyberMed 3.0 is under development to support volumetric models and operations, as cut and suture, and also 
to integrate new devices, new collision detection methods and new collaboration modes.

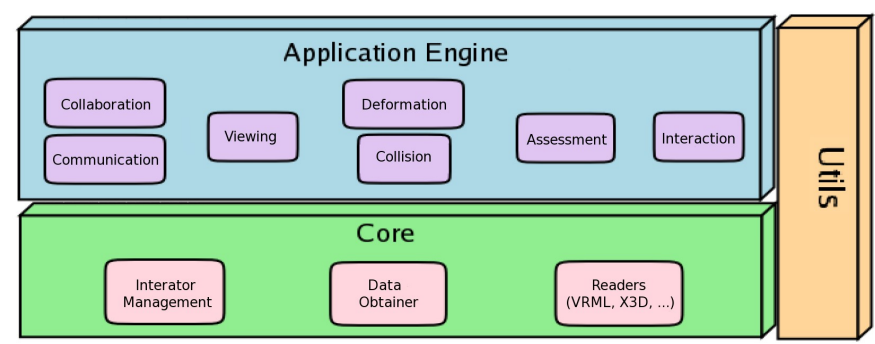

Figure 2. The CyberMed 2.0 framework.

\section{IV.Systems to Support Learning}

Systems have been developed by LabTEVE to support learning in many areas. To solve the problem of different computational configuration in educational labs at universities, the KlabTEVE was developed in 2004: a Linux remasterization from Kurumin distribution for educational purposes. This system could start from $\mathrm{CD}$ and provided automatic network configuration, programming languages $(\mathrm{C} / \mathrm{C}++$, Pascal and JAVA), programming libraries (OpenGL/GLUT), graphical editors (Blender), image processing (XV and GIMP), statistical software (R and OpenStat), OpenOffice and Latex (Kile and LyX), among others functionalities.

The Statistics Calculator [4] was proposed in 2004 as a system for present and distance learning. It consists on an intelligent tool and an interactive environment able to guide the user through an statistical analysis according to the correct steps. This is performed by restrictions analysis of each operation request from user. Such analysis inform users the reasons of their mistakes and helps and stimulates students in their knowledge construction. In 2007 this system received a new visual graphics interface. The same concept was proposed in the Educaview, a system to complement the teaching of image classification. This system follows a pedagogical approach based on Constructivism, as the Statistics Calculator. Educaview uses elements of VR, as interactive 3D visualization, to reinforce concepts and allows students to perform interactive experiments with classification of images with up to three bands. The system contains implementations for the classification methods K-Nearest Neighbor and KMeans [5].

The enhancement of remote experimentation was the focus of development of the remote laboratories with haptics. Remote laboratories allow users to access and interact with real hardware at distance and the use of haptic devices aimed to improve the interaction quality (Figure 3). This project started in 2007 and produced new interfaces with haptics [6]. New studies about the impact of haptics in distance learning based on experimentation for engineering have been conducted since then.

The ability of teacher to develop educational tools by themselves have guided the creation of an architecture to support the visual development of VR applications for nonprogrammers. This architecture, called CyberMedVPS, has produced a system that integrates frameworks for VR and allows production of applications through flowchart representations [7]. As in previous systems, the main idea is offer visual tools to complement and enhance the learning process.

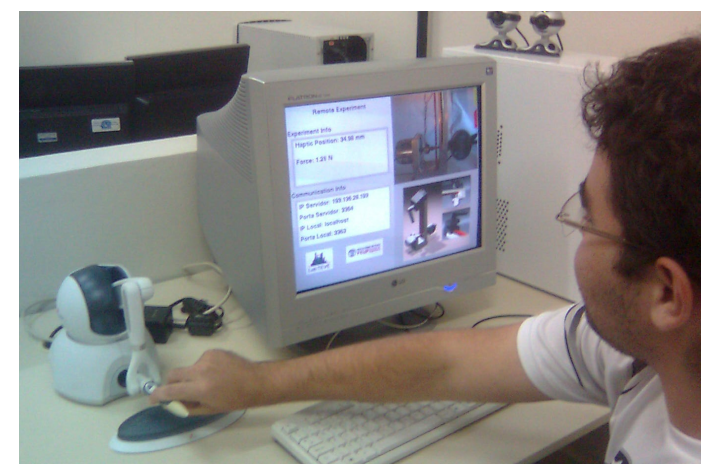

Figure 3. Remote laboratory accessed with haptics.

\section{VR Applications Development}

The first applications developed by LabTEVE were visualizations for several areas as teaching of image classification and a 3D atlas of anatomy based on the VirtWall. After the CyberMed creation, some simulators for training in medicine were developed using it, as the bone marrow transplant simulator [1] and the SITEG for gynecological examination [8]. Those projects allow the partnership of the laboratory team with professionals of the health area whose provided the specific concepts. This interaction has allowed the discussion about validation processes of VR systems for health.

The Virtual Campus was also one of the first projects of LabTEVE and produced 3D mockups of the university campus for navigation. The mockups were expanded in 2008 to connect real and virtual spaces. This project introduced hyperreality concepts and used software and hardware to allow bidirectional control and communication between real and virtual worlds [9].

The project Museu 3I (immersive, interactive, itinerant) produced an infrastructure to quickly publish expositions and allow spreading of information and culture. The project presented two modules to support publication and visiting of $3 \mathrm{D}$ expositions on the web: curator and visitor. Curator module is used to compose collections and to publish the expositions on the web. The visitor module allow users on the web choosing the expositions to be accessed, visualizing them and interacting with the 3D pieces. Nowadays, Museu 3I has two expositions available: Archeology from Paraíba and Orthodontic deformations [10]. The pieces were acquired with a 3D scanner.

Dissemination of culture and information access pointed the development of the Ingá Virtual. It consists on a virtual and multimedia representation of the archeological site of Itacoatiaras do Ingá located in the Paraíba state. The site was modeled in 3D and enriched with multimedia content to virtually presents the place and invite users to know about this important Brazilian archeological site. The project was presented in 2011 for use in PCs and multiprojection systems (Figure 4). 


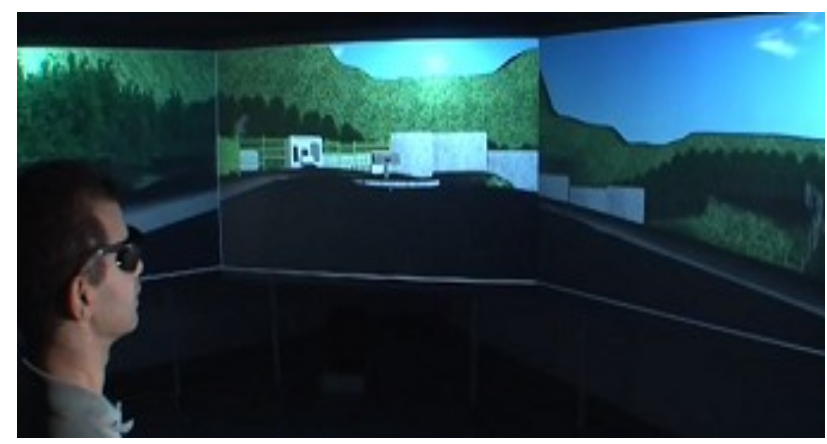

Figure 4. Multiprojection view of the Ingá Virtual project.

\section{VI.Serious Games}

Serious Games aim the development of specific subjects and skills in order to enrich users knowledge through an entertainment approach. Based on that concept, LabTEVE developed games for the learning of Mathematics and Geography for children on the first years of school. Such games were created, modeled and implemented to support different spaces of learning and run in mobile phones, ordinary computers and virtual reality platforms. These games include methodologies that allow the use of intelligence to increase users degree of motivation and challenge. As example, can be mentioned the use of Bayesian Networks and Evidence Theory [11].

The intelligent systems included in serious games can be also used to measure the level of understanding of the concepts and/or patterns followed by the user in a game, as well as to modify the game in progress to include events in order to more accurately measure user's level of expertise. In another way, intelligent systems can be used to reinforce concepts or practices for rehabilitation or professional behavior.

Other serious games were recently launched by LabTEVE, as Motrilha to support speech therapies rehabilitation. Dentistry was the focus of two other serious games: the Adventure in Toothland was developed to inform mothers about good practices for oral health care in babies under three years. This game used an approach based on agents to support decision making in the game intelligence. Also for Dentistry, the Touchbrush game (Figure 5) investigated the use of haptic devices in games and provides an solution for the learning of good practices in tooth brushing [12].

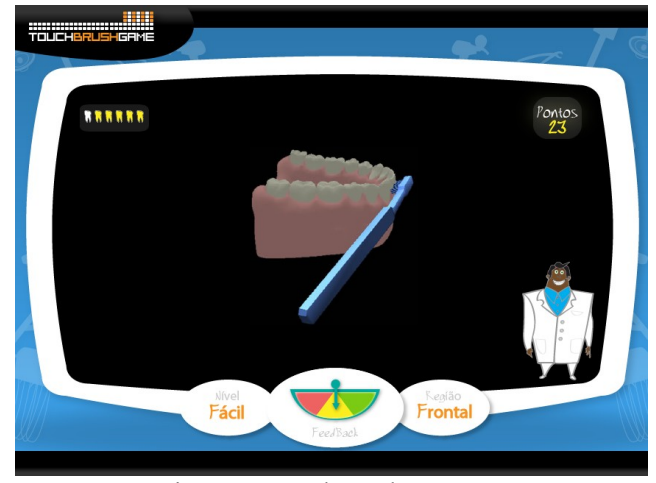

Figure 5. TouchBrush game.

\section{VII.AssesSMENT}

Training assessment based on VR consists on systems which must continuously monitor all user's interactions and compare their performance with predefined expert's classes of performance to recognize user's level of training. Nowadays those systems are evolving of seminal works proposed by Dinsmore et al. [13] in the 90's. Two kinds of training assessment can be found in the literature: single user's assessment system (SUAS) and multiple users' assessment system (MUAS). Basically, SUAS can be divided in off-line and on-line. Off-line SUAS can be defined as methods not coupled to VR systems, whose assessment results are provided some time (which can be minutes, hours or days) after the end of the VR-based training. On the other hand, on-line SUAS are coupled to the training system and collect user data to provide a result of their performance at the end of the simulation [14]. An on-line SUAS works coupled to a VR simulator, as showed in the Figure 6 [15]. A SUAS should be capable to monitor user's interactions with the VR simulator by variables such as position (of user and of instruments used in the VR simulation), touch force and resistance and user's angle of visualization, among others. All the information are sent to the SUAS which analyzes the data and emits, at the end of the training, an assessment report about the user's performance according predefined classes of performance. An on-line SUAS must have low complexity to does not compromise VR simulations performance, but it must have high accuracy to does not compromise the assessment.

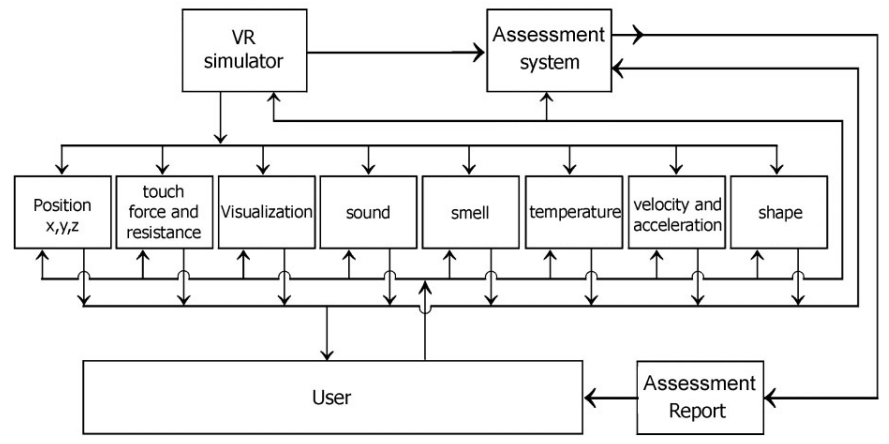

Figure 6. Diagram of a VR simulator with a SUAS. Adapted from [15].

The main differences of VR training systems for multiple users from VR training systems for single users are: increase of complexity, use of clusters of computers or a computer capable to generate realistic multiple views, support to changes in virtual environments for all users, support in assessment system, speed of network for communication among computers without compromising the simulation. Eventually, more than one interaction device, as haptic devices, can be installed in a computer and/or tracking systems for each user in training. A MUAS must be interconnected with all users and must receive from them synchronized information about all variables of interest. A MUAS works as a SUAS (Figure 6), and it should be capable to monitor all interactions among users. In order to reach that, it is necessary to collect information about user's position in the space using tracking systems [2]. User's interactions in the simulation should respect the physics laws 
and train their proper positions in the procedure. To collect some information as force, force feedback, angles and torques, it is necessary to use specific devices to provide them. This information will be collected for each user in training system, as well as for all group, to be used to feed the MUAS. Additionally, synchronization in time and space is necessary for all users to measure interactions among them, to determine the ordering of tasks and to provide details of user's performance [14]. Then, each application will have their own set of relevant variables which will be monitored for each user and also another set of relevant variables which measure interactions among users in the group. All those information is used by MUAS, which analyzes the data and emits, at the end of the training, an assessment report about the individual user's performance according pre-defined classes of performance, as well as, another assessment report about the group performance. There is no classification for MUAS as on-line or off-line, because MUAS are not be able to generate reports immediately after of the end of training session.

In addition to those two assessment types, there are two other: continuous assessment for a single user in sequences of trainings conducted and training assessment executed by Web (used for distance training or distance learning). In the first one, the main focus is use techniques of continuous evaluation to improve trainee performance. Continuous evaluation is a educational methodology used in present and distance learning to help the construction of the knowledge and the cognitive learning. The goal is to construct a profile to help users to understand their difficulties and to improve their performance. The system should be able provide an assessment report and a continuous assessment report, showing the performance of trainee in the last training and in all trainings performed by $\mathrm{him} / \mathrm{her}$, respectively. In the second one, several measures from user interaction in training executed by Web are used as input for an assessment system. Networks limitations should taken into account to design that kind of assessment system.

LabTEVE researchers have worked in design and implementation of assessment systems (SUAS, MUAS, continuous assessment and assessment on Web systems), compatible with the CyberMed framework and, in some cases, the implementation of them on embedded systems.

\section{Future TRENDS}

This decade of activities allowed to consolidate the research group of LabTEVE and establish partnership with several other research groups. Recently, LabTEVE researchers in association with other researchers from UFPB approved projects for acquisition of a computational cluster that should be used, among other purposes, to perform analysis of impacts of global warming in Paraíba state. The laboratory team will be responsible for generating elements of scientific visualization and simulation of 3D models generated from those analysis, using low cost systems as VirtWall ${ }^{\mathrm{TM}}$ or CAVE for 3D visualization.

LabTEVE is part of the Brazilian Institutes of Science and Technology, through the research network Aided Medical Scientific Computing (INCT-MACC), which involves more than 20 Brazilian and international institutions. In addition,
LabTEVE has as research partners several of those institutions for specific projects, as the Faculty of Engineering of Porto (Portugal), in the area of haptics and remote laboratories. Due to the multidisciplinary aspect of its researches, LabTEVE welcomes researchers and students of all areas.

\section{ACKNOWLEDGMENT}

The authors would like to thank all researchers and students of the laboratory that contributed and contribute in the development of new technologies and projects. The authors also would like to thank support agencies as CNPq, CAPES, FINEP and the Brazilian Ministry of Culture.

\section{REFERENCES}

[1] D.F.L. Souza, I.L.L. Cunha, L.C. Souza, R.M. Moraes, L.S. Machado, "Development of a VR Simulator for Medical Training Using Free Tools: A Case Study", Proc. Symposium on Virtual and Augmented Reality, pp. 100105. Brazil. 2007.

[2] D.F.L. Souza, L.S. Machado, R.M. Moraes. "Integration of Tracking Systems for the Development of Virtual Reality Application". IEEE Latin America Transactions 8(6):714-721, 2010.

[3] R.M. Moraes and L.S. Machado, "Development of Medical training Systems with Integration of Users Assessment". Book Chapter. In: Jae-Jin Kim (Ed.), Virtual Reality, Chapter 15. Croatia: Intech. 2011.

[4] R.G.S. Gomes, L.S. Machado, R.M. Moraes. "The Calculadora Estatística for Present and Distance Learning". International Conference on Teaching Statistics, 2006, Salvador. Proceedings of ICOTS, 2006.

[5] G.P. Dias, L.S. Machado, R.M. Moraes. "Um Sistema Baseado em Construtivismo e Realidade Virtual para Apoio ao Ensino de Classificadores de Imagens". International Conference on Engineering and Computer Education, 2009, Buenos Aires. Proceedings of ICECE 2009, 2009.

[6] L.S. Machado, T.K.L. Costa, M.T. Restivo, R.M. Moraes. "Improving Interaction in Remote Laboratories Using Haptic Devices". REV2007 Conference, 2007, Porto. Proceedings of REV International Conference. Porto: Kassel University Press, 2007. p. 1-5.

[7] A.M. Morais, L.S. Machado. "CyberMedVPS: Visual Programming for Development of Simulators". Studies in Health Technology and Informatics, 163: 386-388, 2011.

[8] L.S. Machado, M.C.O. Valdek, R.M. Moraes. "On-Line Assessment System for a Training System with Multiple Stages Based on Virtual Reality". Journal of Multiple-Valued Logic and Soft Computing 14: 511-524, 2008.

[9] T.K.L. Costa, B.R.A. Sales, R.M. Moraes, J.A.G. Lima, L.S. Machado. Real Environments Management Through Virtual Campus. Int. Conference on Engineering and Technology Education (Intertech), 2008, Santos. p. 534-538.

[10] E.L. Falcão, H.R. Rocha Neto, L.S. Machado. "Multiprojeção por Clusterização de Visualização de Ambientes Virtuais com o Xj3D". Symposium on Virtual and Augmented Reality, 2011.

[11] R.M. Moraes, L.S. Machado, F.L.S. Nunes, R.M.M. Costa. "Serious Games and Virtual Reality for Education, Training and Health". Book Chapter. Cruz-Cunha (Org.) Serious Games as Educational, Business, and Research Tools: Development and Design. IGI Global, 2012 (in press).

[12] A.M. Morais, H.F. Rodrigues, L.S. Machado, A.M.G. Valença. "Planning Serious Games: Adapting Approches for Developmen"t. Lecture Notes in Computer Science 6249:385-394, 2010.

[13] M. Dinsmore, "Virtual reality simulation: training for palpation of subsurface tumors". Master's Thesis, Department of Mechanical and Aerospace Engineering, Rutgers University, October, 1996.

[14] L.S. Machado, R.M. Moraes, "Multiple Assessment for Multiple Users in Virtual Reality Training Environments". Lecture Notes in Computer Science 4756: 950-956. Springer, 2007.

[15] L.S. Machado, R.M. Moraes, R.M., Intelligent Decision Making in Training Based on Virtual Reality. Book Chapter. Da Ruan. (Org.) Computational Intelligence in Complex Decision Systems, Chapter 4. Paris: Atlantis Press. 2010. 